Bulletin of the AAS • Vol. 54, Issue 1 (Obituaries, News \& Commentaries, Community Reports)

\title{
Richard (Dick) A. McCray
}

The Colorado Arts \& Sciences Magazine Staff

Published on: Mar 01, 2022

DOI: $10.3847 / 25 c 2 c f e b .283 b 494 e$

License: Creative Commons Attribution 4.0 International License (CC-BY 4.0). 
Richard Alan (Dick) McCray, a leading authority on supernova gas dynamics and science education, died on Tuesday, October 26, 2021. He was 83.

The explosion of Supernova 1987A illuminated the scientific career of University of Colorado Boulder astrophysicist Richard Alan (Dick) McCray. That light continued to shine until his passing on October 26, 2021.

"Dick McCray was a luminary in our field and in our department," said Nils Halverson, professor and chair of the University of Colorado (CU) Boulder Department of Astrophysical and Planetary Sciences. "He will be missed."

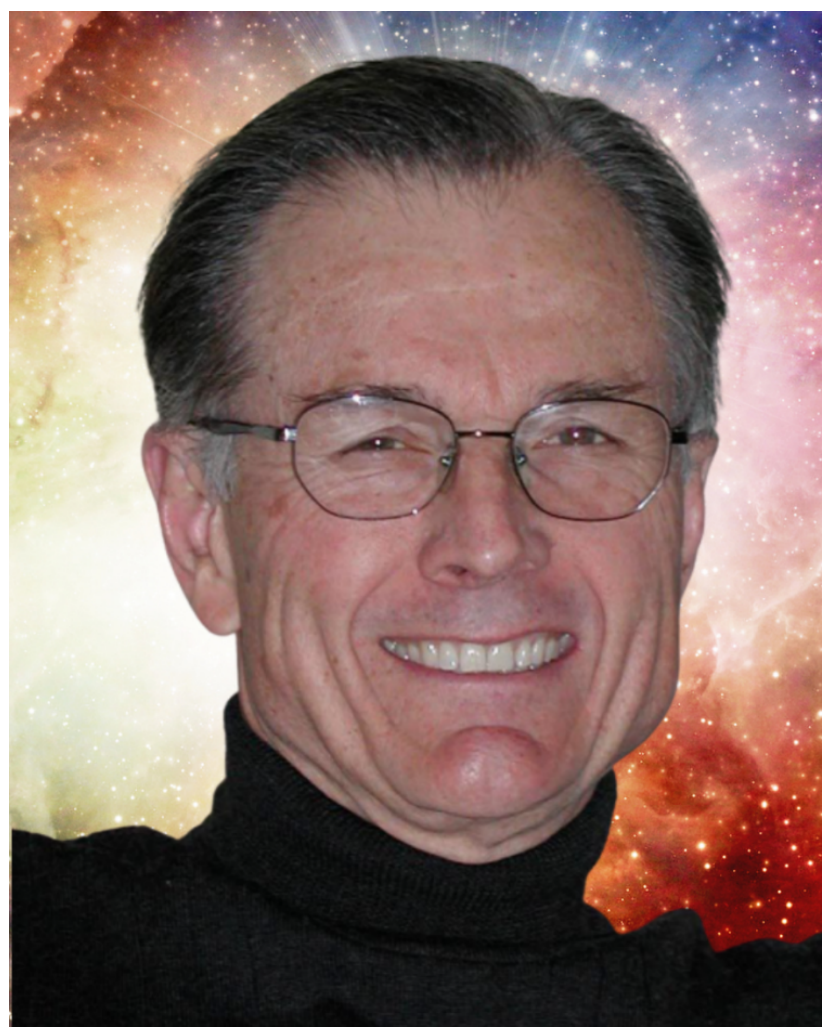

Born on Nov. 24, 1937, in Los Angeles, McCray received his B.S. in physics from Stanford University in 1959 and his Ph.D. in physics in 1967 from the University of California, Los Angeles. He was a research fellow at Caltech and then an assistant professor at Harvard University. He spent most of his career on the faculty in the Department of Astrophysical and Planetary sciences at CU Boulder.

In 2004 he retired as the George Gamow Distinguished Professor of Astrophysics. He was also a visiting scholar at the NASA Goddard Space Flight Center, at Beijing University and Nanjing University, at the Space Telescope Science Institute, at Columbia University, and at the University of California, Berkeley.

Shortly after the initial discovery of Supernova 1987A (SN1987A) in February 1987, he and colleagues Mike Shull and Peter Sutherland predicted that "in several months, we may witness a spectacular display of X-rays and UV emission lines and continuum, as the supernova envelope expands to reveal the inner debris of the explosion." The prediction was confirmed in September 1987 by X-ray observations with the Japanese Ginga (ASTRO-C) X-ray astronomy satellite. McCray continued theoretical and multiwavelength observational studies of SN1987A for the next 20 years. 
In 1990, he and colleague Ding Luo predicted that supernova debris would strike circumstellar matter by 2006, giving rise to a rapidly brightening source of X-rays, infrared and radio emission. The predicted brightening indeed occurred, starting in 2002, and continuing for roughly a decade, resulting in the now-famous "ring of pearls."

McCray was particularly interested in interstellar gas dynamics and the theory of cosmic X-ray sources. He researched the formation of interstellar bubbles by stellar winds and 'super-bubbles' formed by supernova explosions in galactic gas disks. His research on cosmic X-ray sources dealt with, among other topics, the mechanism of the transformation of X-ray emission in the optical and UV range for neutron stars and black holes. In addition to theoretical calculations and computer simulations, he made observations using the Hubble Space Telescope, the Chandra X-ray Observatory, and the Atacama Large Millimeter Array. McCray was also a lifelong learner; he spoke Mandarin and was an avid student of Chinese history and culture.

McCray was a Guggenheim Fellow, member of the National Academy of Sciences and a Fellow of the American Association for the Advancement of Science. In 1990 he received the Dannie Heineman Prize for Astrophysics, and in 2002 he was awarded the National Science Foundation Director's Award for Distinguished Teaching Scholars.

In the 1980s he served as chair of the Joint Institute for Laboratory Astrophysics (JILA), then chair of the newly created Center for Astrophysics and Space Astronomy at CU Boulder.

Beyond his scientific achievements, McCray was deeply engaged in developing innovative pedagogy for undergraduate STEM education. In 2001, he, School of Education Professor Valerie Otero, and other CU Boulder colleagues co-founded the Learning Assistant (LA) model, which has been instrumental in transforming instruction to be more inclusive, leading to better outcomes and in inspiring undergraduate STEM majors to pursue careers as K-12 teachers.

He and his CU Boulder colleagues won the American Physical Society's 2019 Excellence in Physics Education Award for this effort. The LA model has since been imitated internationally, throughout the United States and in 28 countries. The LA model has been described as one of the pillars of campus excellence. As Otero recalls: "Dick was my mentor and my collaborator. Instead of me attending a minority program for at-risk faculty when I first started here in 2001, all he had to say was, 'Wanna collaborate? I need your expertise.' The retention of a first-generation Hispanic, first- 
generation college student, woman in physics was largely due to those words and his follow through on them."

McCray married Sandra Broomfield in 1960. After her passing in 2013, he married Susan Bloch in 2016. He passed into the Lux Eterna that was his lifelong passion on October 26, 2021, and is survived by two children (The Rev. Julia McCray-Goldsmith and Carla McCray) and two grandchildren (Amos McCray-Goldsmith and Aaron McCray-Goldsmith), all residents of the San Francisco Bay Area.

He was an athlete: a swimmer and squash player. He and his family traveled extensively and not always prudently, according to the women who loved him. He was an avid amateur pilot, and his children remember some "pretty wild" landings at rural airfields ... but the sky was always his great delight.

Gifts in honor of Dick McCray, to equip grandson Aaron's inner-city high school physics classroom, may made through Trinity Episcopal Cathedral, 81 N. 2nd St, San Jose, California 95113 (note "McCray Memorial” in the memo), or donate online.

Adapted and reproduced with permission from The Colorado Arts \& Sciences Magazine, a publication of The University of Colorado:

https://www.colorado.edu/asmagazine/2021/11/04/astrophysicist-stem-educationpioneer-richard-mccray-dies-83 